\title{
Aplicaciones publicitarias para móvil: conocimiento, actitudes, motivos de uso y valoración por parte de los adolescentes españoles
}

\author{
Silvia SANZ BLAS ${ }^{1}$ \\ Universitat de València \\ José MARTÍ PARREÑO ${ }^{2}$ \\ Universidad Europea de Madrid \\ Carla RUIZ MAFÉ ${ }^{3}$ \\ Universitat de València
}

\begin{abstract}
RESUMEN:
La publicidad móvil ha ido captando la atención de los anunciantes en los últimos años convirtiéndose en un sector emergente de la actividad publicitaria actual. Fruto de este interés, y del propio dinamismo del sector, los anunciantes han ido experimentando con numerosas estrategias y herramientas, desde los SMS y MMS hasta el bluetooth o los códigos QR, con el objetivo de ser más eficaces a la hora de desarrollar sus comunicaciones de marketing. Las aplicaciones publicitarias para móviles son la penúltima de las herramientas que el medio pone a disposición de los anunciantes. El exponencial crecimiento en el uso de aplicaciones para móviles por parte de los usuarios parece augurar un buen rendimiento de esta herramienta en su uso publicitario. Sin embargo, expectativas previas no cumplidas en el caso de otras herramientas así como la propia evolución del comportamiento de los consumidores con el medio, sugiere abordar con cautela las expectativas generadas en torno a las aplicaciones publicitarias para móviles. Por tanto, el presente trabajo exploratorio tiene como objetivo principal responder a una serie de cuestiones de investigación que permitan detectar actitudes y percepciones de los consumidores españoles hacia las aplicaciones para móviles en general y hacia las aplicaciones publicitarias para móviles en particular con el fin de evaluar el interés de la herramienta como soporte de las comunicaciones de marketing.
\end{abstract}

PALABRAS CLAVE: marketing móvil; publicidad móvil; aplicaciones para móviles; actitud hacia la publicidad.

TITLE: Mobile Advertising Applications: Spanish Teenagers' Knowledge, Attitudes and Motives of Use.

ABSTRACT:

Mobile advertising has gained momentum among advertisers during the last years becoming an emergent sector in nowadays advertising practise. As a consequence of this advertisers' interest - and also the dynamism of the market - advertisers have been experimenting with quiet of strategies and tools - from SMS and MMS to Bluetooth or QR Codes - as a means of achieving a better effectiveness while developing their marketing communications. Mobile advertising applications are one of the last of such tools that advertisers can use within mobile phones. The increasing growth of consumer's use of mobile applications seems to assure quite a good perfor-

1 Profesora Titular del Departamento de Comercialización e Investigación de Mercados. Facultad de Economía. Universitat de València. E-mail: silvia.sanz@uv.es

2 Profesor del Departamento de Empresa. Universidad Europea de Madrid. E-mail: jose.marti@uem.es

3 Profesora Titular del Departamento de Comercialización e Investigación de Mercados. Facultad de Economía. Universitat de València. E-mail: carla.ruiz@uv.es 
mance of this advertising tool. Despite this, previous expectations not achieved when using other mobile advertising tools - as well as the evolving role of consumer behaviour within the medium - suggest to be cautious on mobile advertising applications' effectiveness. This exploratory paper has as a main objective to answer some research questions that allow us to detect Spanish consumers' attitudes and perceptions of mobile applications in general - as well as mobile advertising applications - to evaluate the interest of such a tool as a means of marketing communications.

KEY WORDS: mobile marketing; mobile advertising; mobile applications; attitude toward advertising.

\section{Introducción}

Con cerca de 5.900 millones de suscripciones y una tasa de penetración a nivel mundial del $87 \%$, la telefonía móvil se ha convertido en la más ubicua de las tecnologías $^{4}$. Los teléfonos móviles, en sus inicios simples dispositivos que ofrecían servicios de voz y texto, son actualmente complejos dispositivos multimedia con los que los usuarios pueden tomar y enviar vídeos y fotografias, navegar por Internet, descargarse canciones o jugar a videojuegos online. Características del medio como la personalización, la localización, la ubicuidad o la interactividad, han situado a la publicidad móvil en un lugar estratégico dentro de los servicios relacionados con la comunicación publicitaria $^{5}$. De hecho se espera que la publicidad a través del móvil ocupe el segundo lugar de inversión que más crezca en los próximos cinco años dentro de los denominados medios alternativos (tan sólo por detrás del consumer-generated media) ${ }^{6}$. En este mismo sentido, las previsiones del sector en España son optimistas puesto que se espera alcanzar los 90 millones de euros de inversión en marketing móvil en 2012, lo que supone un crecimiento medio del $53 \%{ }^{7}$. Así pues no parece extraño que los anunciantes muestren cada vez un mayor interés en el uso de un medio que les ofrece una comunicación directa con los consumidores, cuyas respuestas por parte del consumidor pueden medirse en tiempo real y que además ofrece una amplia variedad de formatos $\mathrm{y}$ estrategias $^{8}$.

4 International Telecommunication Union (2011): «The World in 2011: ICT Facts and Figures», disponible en: http://www.itu.int/ITU-D/ict/material/FactsFigures2011.pdf (fecha de consulta: 30/07/11).

5 ZHANG, J. y MAO, E. (2008): «Understanding the Acceptance of Mobile SMS Advertising among Young Chinese Consumers», Psychology \& Marketing, 25 (8), 787-805; OKAZAKI, S., Li, H. y HIROSE, M. (2009): «Consumer privacy concerns and preference for degree of regulatory control: A study of mobile advertising in Japan», Journal of Advertising, 38 (4), 63-77.

${ }^{6} \mathrm{PQ}$ MEDIA (2008): «Alternative media forecast: 2008-2012», disponible en: http://www.pqmedia.com/alternative-media-forecast-2008.html (fecha de consulta: 12/05/09)

7 MMA (MOBILE MARKETING ASSOCIATION) (2010): «3er Estudio de Inversión en Marketing y Publicidad Móvil en España 2010», disponible en: http://recursos.anuncios.com/files/387/64.pdf (fecha de consulta: 19/04/11)

8 SANZ, S., RUIZ, C. Y MARTí, J. (2011): «Situación actual de las comunicaciones de marketing mediante telefonía móvil», ponencia presentada en el XXV Congreso Anual de la Academia Europea de Economía y Dirección de Empresa, Actas del Congreso, Valencia 8, 9 y 10 de junio. 
Las aplicaciones para móviles (mobapps) han pasado a engrosar la lista de potenciales soportes que pueden ser utilizados por los anunciantes para alcanzar sus objetivos publicitarios. Con un volumen de negocio estimado cercano a los 30.000 millones de dólares en $2013^{9}$, las Application Stores representan un sector emergente en el mercado de la telefonía móvil. La App Store de Apple, por ejemplo, ofrece más de 225.000 aplicaciones, tanto gratuitas como de pago, de las que ya se han realizado más de 6.500 millones de descargas. El resto de fabricantes de smartphones también tratan de monetizar este prometedor segmento de mercado lanzando sus propias tiendas de aplicaciones. Es el caso de Nokia con su Ovi Store, abierta en mayo de 2009, que da servicio a más de 100 dispositivos móviles distintos y a través de la que se realizan cerca de 1,5 millones de descargas al día ${ }^{10}$. Otras tiendas de aplicaciones son Sony Ericsson Store, Samsung Store, Android Market de Google y Market Place de Microsoft. Unas App Store en las que se ofrece todo tipo de contenidos móviles, desde tonos de llamada hasta canciones y tráileres cinematográficos, videojuegos o aplicaciones que permiten reconocer qué canción está sonando en la radio.

Sin embargo, expectativas previas no cumplidas relacionadas con el uso de otras herramientas de la publicidad móvil animan a ser cautos ante las enormes posibilidades que parecen ofrecer las aplicaciones para móviles a la comunicación publicitaria. En este sentido, el presente trabajo exploratorio tiene como objetivo principal responder a una serie de cuestiones de investigación que permitan detectar actitudes y percepciones de los consumidores españoles hacia las aplicaciones para móviles en general y hacia las aplicaciones publicitarias para móviles en particular con el fin de evaluar el interés de la herramienta como soporte de las comunicaciones de marketing. Para ello, en primer lugar se conceptualizará la publicidad móvil y se analizarán las características que presentan las aplicaciones publicitarias para móvil. A continuación se plantearán las cuestiones de investigación y se describirá la metodología empleada en el estudio. Posteriormente se realizará el análisis de los resultados y se expondrán las conclusiones derivadas de ellos. Finalmente, se apuntarán las principales implicaciones directivas, las limitaciones del estudio y las futuras líneas de investigación.

\section{Concepto de publicidad móvil}

El término publicidad móvil ha sido definido académicamente desde diferentes perspectivas que abarcan desde el marketing móvil hasta el marketing inalámbrico o la publicidad inalámbrica, centrándose la mayoría de las definiciones en la propia tecnología utilizada ${ }^{11}$. Desde una perspectiva comunicativa, las comunicaciones de marke-

9 GARTNER (2009): «Dataquest Insight: Application Stores; The Revenue Opportunity Beyond the Hype», disponible en: http://www.gartner.com/resId=1257213 (fecha de consulta: 19/08/10)

10 ASIMELEC. (2010): «Informe 2010 del macrosector TIC en España».

11 LepPÄniEMi, M., SinISALO, J. y KARJALUOTO, H. (2006): «A review of mobile marketing research, International Journal of Mobile Marketing», 1 (1), 30-40. 
ting móvil han sido conceptualizadas por la Mobile Marketing Association como "el conjunto de acciones que permite a las empresas comunicarse y relacionarse con su audiencia de una forma relevante e interactiva a través de cualquier dispositivo o red móvil" ${ }^{12}$. Entre este conjunto de acciones se encuentran el envío de mensajes a través de SMS, el uso de formatos gráficos o display, el marketing en buscadores a través de Internet móvil, el bluetooth, el couponing o el uso de aplicaciones y contenidos (entre los que se encuentra el advergaming $)^{13}$.

Como principales ventajas de la puesta en marcha de una campaña de publicidad móvil se han señalado: a) alcance y versatilidad: así por ejemplo, los SMS permiten pasar del marketing masivo al marketing one to one, b) permanencia y alcance viral: los SMS permanecen en el buzón de mensajes del cliente hasta que son borrados, pudiendo el usuario reenviarlo si lo considera de interés, c) ubicuidad e inmediatez: la tecnología permite personalizar el instante en que el usuario recibe nuestro mensaje, d) interactividad: el mismo canal utilizado por la empresa para contactar con el cliente puede ser usado por éste para contestar y ponerse en contacto con la marca, e) rapidez y adaptabilidad: el tiempo necesario para poner en marcha una campaña es mínimo, y el feedback inmediato, permitiendo realizar cambios cuando es necesario, f) conveniencia: los SMS permiten acceder al cliente donde esté y a cualquier hora, y g) geolocalización: la tecnología actual permite personalizar el lugar donde el cliente recibe el mensaje, y también lanzarlo sólo si el cliente se encuentra en una determinada ubicación ${ }^{14}$.

\section{Aplicaciones para móviles y aplicaciones publicitarias}

Las aplicaciones para móviles consisten en un software (en muchos casos gratuito o bajo modelo freemium $^{15}$ ) que se ejecuta desde el dispositivo móvil y permite al usuario

12 MMA (Mobile MARKETING AsSOCIATION) (2010): «3er Estudio de Inversión en Marketing y Publicidad Móvil en España 2010», p. 7. Disponible en: http://recursos.anuncios.com/files/387/64.pdf (fecha de consulta: 19/04/11)

13 Ibídem.

14 Haggirian, P., Madlberger, M. y TAnuskova, A. (2005): «Increasing Advertising Value of Mobile Marketing- An Empirical Study of Antecedents», Proceedings of the 38th Hawaii International Conference on System Sciences. 3-6 enero, Hawai; INTERACTIVE ADVERTISING BUREAU (IAB) (2009): «Estudio de Internet e interactividad en móviles $y$ otros dispositivos portátiles», disponible en: http://www.iabspain.net/ver.php?mod=noticias\&identificador=40 (fecha de consulta: 09/05/10); KARGIN, B., NuRi BASOGlu, N. Y TUGRUL DAiM, T. (2009): «Factors affecting the adoption of mobile services», International Journal of Services Sciences, Vol. 2, Nº1, pp. 29-52; SCHARL, A., DiCKINGER, A. Y MURPHY, J. (2005): «Diffusion and success factors of mobile marketing», Electronic Commerce Research and Applications, 4 (2), 159-173; Shankar, V. y Balasubramanian, S. (2009): «Mobile Marketing: A Synthesis and Prognosis», Journal of Interactive Marketing, 23, 118-129; ZHANG, J. Y MAO, E. (2008): «Understanding the Acceptance of Mobile SMS Advertising among Young Chinese Consumers», Psychology \& Marketing, 25 (8), 787-805.

${ }_{15}$ Este modelo de negocio se basa en una gratuidad inicial que es combinada con pequeños micropagos por parte del consumidor si quiere acceder a determinados servicios extras de la aplicación (Adelantado y Martí, 2010). 
del teléfono desarrollar determinadas tareas. La extensión en su uso se debe a las múltiples funciones que pueden desarrollar, incluyendo distintas interfaces para el uso de servicios básicos de telefonía o mensajería, así como servicios más avanzados de vídeo, juegos o herramientas para la descarga y lectura de blogs, etc ${ }^{16}$. Estas aplicaciones también pueden ser utilizadas como herramienta publicitaria para desarrollar diferentes acciones. De hecho, las aplicaciones móviles generaron en 2010 el 32\% de la inversión realizada en publicidad móvil. Una de sus principales ventajas es que permiten al anunciante interactuar y crear un contacto directo con los clientes o con el público objetivo al que van dirigidas las comunicaciones de marketing ${ }^{17}$. Además, suponen una oportunidad para las marcas de interactuar con sus audiencias e impactarlas de forma no intrusiva ya que es el propio usuario quien se descarga voluntariamente la aplicación a su teléfono móvil. Otra de sus ventajas es su viralidad, ya que desde la misma aplicación se puede enviar recomendaciones de descarga a amigos y conocidos. Además se ha detectado que la publicidad mediante este tipo de formatos incrementa el recuerdo de marca y motiva la implicación con la marca y la compra ${ }^{18}$. Telepizza es una de las marcas que ha desarrollado una aplicación que permite realizar pedidos directamente desde el teléfono móvil del usuario. Zippo es otra de las marcas que ha desarrollado aplicaciones publicitarias para móviles (en este caso para el iPhone). En muchos casos estas aplicaciones publicitarias no dejan de ser pequeños divertimentos como el mechero virtual Zippo que el usuario puede "encender" tocando la pantalla de su teléfono móvil (la aplicación también permitía al usuario "jugar" con la llama, una vez encendido el mechero, soplando sobre la pantalla y provocando así cambios en su dirección e intensidad). En otros casos, se han desarrollado aplicaciones publicitarias de una mayor complejidad. Fiat, por ejemplo, utilizó una compleja aplicación, llamada Fiat Street Evo, para promocionar el Punto Evo. Esta aplicación podía descargarse tanto para iPhone como para dispositivos bajo plataforma Android. El usuario debía descargarse la aplicación, registrarse y captar con su móvil las señales de tráfico que iba encontrando en la ciudad. Cada señal se asociaba con una característica del modelo de Fiat. Por ejemplo, si el móvil captaba una señal de Stop, la aplicación informaba de que el Fiat Punto Evo cuenta con frenos ABS. Si se trataba de una señal de curvas, la aplicación informa de que este vehículo tiene el sistema de faros con "cornering" para garantizar el máximo control en cada curva. La aplicación (utilizando el GPS del móvil) también podía guiar al usuario al concesionario más cercano para que pudiera conocer al nuevo Punto Evo en persona y conducirlo. Además la acción se completó con una promoción de premios entre los usuarios que habían utilizado la aplicación (entra-

16 MMA (Mobile Marketing Association) (2008): «Mobile Applications», disponible en: http://mmaglobal.com/mobileapplications.pdf(fecha de consulta: 14/07/09).

17 MMA (MOBILE MARKETING ASSOCIATION) (2010): «3er Estudio de Inversión en Marketing y Publicidad Móvil en España 2010», Disponible en: http://recursos.anuncios.com/files/387/64.pdf (fecha de consulta: 19/04/11)

18 Xu, D.J., Liao, S.S. y Li, Q. (2008): «Combining empirical experimentation and modelling techniques: A design research approach for personalized mobile advertising applications», Decision Support Systems, 44 (3), 710-724. 
das para el Gran Premio de Monza de F1, iPads, bolsas de viaje, camisetas, neveras...). Los premios se escondían tras cada señal captada utilizando la mecánica de momento ganador (swepstake).

\section{Cuestiones de investigación}

Este trabajo exploratorio se centra en cuatro áreas de interés principales a la hora de determinar el interés de la aplicaciones publicitarias para móvil como herramienta de las comunicaciones publicitarias: a) conocimiento de las aplicaciones publicitarias para móvil por parte de los consumidores, b) actitud de los consumidores hacia las aplicaciones publicitarias para móvil, c) motivos de uso de las aplicaciones publicitarias para móvil por parte de los consumidores, y d) valoración de las aplicaciones publicitarias para móvil por parte de los consumidores.

A continuación, se realiza la revisión de la literatura que permite dar soporte a las cuestiones de investigación planteadas en el presente trabajo:

\subsection{Conocimiento de las aplicaciones publicitarias para móvil}

La primera cuestión de investigación se dirige directamente a conocer el grado de consciencia o conocimiento de las aplicaciones publicitarias para móvil por parte de los adolescentes españoles. El conocimiento del objeto publicitario es prioritario en todos los modelos de jerarquía de los efectos ${ }^{19}$ como paso previo a la formación posterior de actitudes y acciones comportamentales hacia dichos estímulos. Es decir, no puede haber reconocimiento, actitud o comportamiento hacia un objeto cuya existencia se desconoce. En este sentido, se plantea la siguiente cuestión de investigación:

Cuestión de investigación 1: ¿conocen los adolescentes españoles las aplicaciones publicitarias para móvil?

\subsection{Actitud hacia las aplicaciones publicitarias para móvil}

Por otra parte, la actitud hacia el anuncio se ha considerado tradicionalmente una medida de eficacia publicitaria ${ }^{20}$, en especial al considerar los efectos que ejerce la

19 Strong, E. K. (1925): The Psychology of Selling, Nueva York, MacGraw-Hill; LAVIDGE, R. J. Y STEINER, G. A. (1961): «A Model for Predictive Measurements of Advertising Effectiveness», Journal of Marketing, 24, 59-62; MCGuIRE, W. J. (1978): «An Information Processing Model of Advertising Effectiveness», en Davis, H.L y Silk, A.J. (eds.) Behavioral and Management Science in Marketing, Nueva York, Ronald/Wiley.

20 LuTZ, R.J. (1985): «Affective and Cognitive Antecedents of Attitude Toward the Ad: A Conceptual Framework», Alwitt, L. y Mitchell, A. (Eds.), Psychological Processes and Advertising Effects, Hillsdale (NJ), Lawrence J. Eribaum, 45-65; MACKENZIE, S. C., LuTZ, R. J. y BelCH, G.E. (1986): «The Role of Attitude toward the Ad as a mediator of Advertising Effectiveness: A Test of Competing Explanations», Journal of Marketing Research, 23, (mayo), 130-143; BATRA, R. Y RAY, M. L. (1986): «Affective Respons- 
actitud hacia el anuncio sobre la actitud hacia la marca ${ }^{21}$. Esto es debido, entre otros factores, a que se ha detectado que se puede producir una transferencia directa de la actitud hacia el anuncio sobre la actitud hacia la marca a través de mecanismos de condicionamiento clásico ${ }^{22}$. En este sentido, resulta importante conocer cuál es la actitud de los usuarios hacia las aplicaciones publicitarias para móvil, conceptualizadas aquí como anuncios, para poder evaluar su eficacia como formato publicitario. En este sentido, se plantea la siguiente cuestión de investigación:

Cuestión de investigación 2: ¿cuál es la actitud de los adolescentes españoles hacia las aplicaciones publicitarias para móvil?

\subsection{Motivos de uso de las aplicaciones publicitarias para móvil}

Por otra parte, el conocimiento del motivo por el cual los usuarios deciden descargar y utilizar aplicaciones publicitarias para móvil puede aportar importante información que permita incrementar la eficacia de las acciones publicitarias desarrolladas mediante aplicaciones publicitarias para móvil. En este sentido, la teoría de usos y gratificaciones $^{23}$ aporta un sólido marco de trabajo. Esta teoría asume que los consumidores seleccionan y usan conscientemente ciertos medios y contenidos que gratifican necesidades específicas tanto de información como de entretenimiento o sociales y puede utilizarse para diseñar comunicaciones de marketing que resulten más relevantes y, por tanto, más eficaces para los consumidores. En este sentido, se plantea la siguiente cuestión de investigación:

Cuestión de investigación 3: ¿cuál es el motivo por el que los adolescentes españoles se descargan y utilizan aplicaciones publicitarias para móvil?

es Mediating Acceptance of Advertising》, Journal of Consumer Research, Vol. 13, (septiembre), 234-249; AaKer, D. A., Stayman, D. M. y VezINA, R. (1988): «Identifying Feelings Elicited by Advertising», Psychology and Marketing, 5 (primavera), 1-16; GEUENS, M. Y DE PELSMACKER, P. (1998): «Feelings Evoke by Warmth, Eroticism and Humour in Alcohol Advertissments», Journal of Consumer and Market Research, disponible en http://www.amsreview.org/articles/geuens01-1998.pdf (fecha de consulta: 29/07/07).

21 BATRA, R. Y RAY, M. L. (1986): «Affective Responses Mediating Acceptance of Advertising», Journal of Consumer Research, Vol. 13, (septiembre), 234-249; Mitchell, A. A. Y Olson, J. C. (1981): «Are Product Attribute Beliefs the Only Mediator of Advertising Effects on Brand Attribute?», Journal of Marketing Research, 18, (agosto), 318-332; MOORE, D. L. Y HuTCHINSON, J. W. (1983): «The Effects of Ad Affect on Advertising Effectiveness», en Bagozzi, R. y Tybout, A. (eds.), Advances in Consumer Research, vol. 10, Ann Arbor (Michigan), Association for Consumer Research, 526-531; PARK, C. W. Y YounG, S. M. (1986): «Consumer Response to Television Commercials: The Impact of Involvement and Background Music on Brand Attitude Formation», Journal of Marketing Research, 23, (febrero), 11-24; SHIMP, T. A. (1981): «Attitude toward the Ad as Mediator of Consumer Brand Choice», Journal of Advertising, 10 (2), 9-15.

22 Kim, J. Allen, C. T y Kardes, F. R. (1996): «An Investigation of the Mediational Mechanisms Underlying Attitudinal Conditioning», Journal of Marketing Research, 33 (agosto), 318-328.

23 KATZ, E., HAAs, H. y GuREvitch, M. (1973): «On the Use of the Mass Media for Important Things», American Sociological Review, 38 (2), 164-181. 


\subsection{Valoración de las aplicaciones publicitarias para móvil}

La última cuestión de investigación se dirige a medir la valoración que efectúan los adolescentes españoles sobre las aplicaciones publicitarias para móvil. De forma general, la publicidad en el móvil no está bien valorada por el consumidor, quizás debido a su percepción como herramienta intrusiva vinculada a la falta de control por parte del usuario $^{24}$. Esa valoración puede cambiar si analizamos la publicidad que se lleva a cabo a través de las aplicaciones, ya que su acceso es totalmente voluntario y controlado en todo momento por la persona. Se trata de un formato publicitario que se encuentra todavía en fase inicial y de experimentación, por lo que todavía no existen estudios que hayan analizado cómo es valorado por el consumidor. Por este motivo, consideramos importante conocer en el presente estudio cómo son valoradas las aplicaciones publicitarias para móvil.

Cuestión de investigación 4: ¿cómo valoran los adolescentes españoles las aplicaciones publicitarias para móvil?

\section{Metodología}

Nuestro público objetivo está formado por jóvenes adolescentes usuarios de telefonía móvil. En total se ha obtenido una muestra válida de 274 jóvenes, de edades comprendidas entre 14 y 16 años, estudiantes de segundo, tercero y cuarto de E.S.O. Del total de la muestra un $58,5 \%$ son hombres y un $41,5 \%$ mujeres. El trabajo de campo fue llevado a cabo durante los meses de Junio y Julio de 2011. Se ha seleccionado a los jóvenes adolescentes como muestra objeto de estudio, debido a que investigaciones previas afirman que son los consumidores más jóvenes los que realizan un uso más intenso de la tecnología en general y de la telefonía móvil en particular ${ }^{25}$. De hecho, la telefonía móvil se ha convertido en un elemento esencial del estilo de vida de la juventud convirtiendo a los teléfonos móviles en algo indispensable para los jóvenes ${ }^{26}$. Además, los adolescentes se han convertido es un público de interés preferente para los

24 Tsang, M.M., Ho, S-C. Y LiAnG, T-P. (2004): «Consumer attitudes toward mobile advertising: An empirical study», International Journal of Electronic Commerce, 8 (3), 65-78; WALDT, REBBELLO T.M. Y Brown W.J. (2009): «Attitude of Young Consumers toward SMS", African Journal of Business Management», 3 (9), 444-452; XU, D.J.J. (2006): «The influence of personalization in affecting consumer attitudes toward mobile advertising in China», Journal of Computer Information Systems, 47 (2), 9-19.

25 CONECTA (2011): «6 $6^{\circ}$ observatorio de tendencias NOKIA: los jóvenes, los móviles y la tecnología», disponible en:

http://www.conectarc.com/Articulos\%20y\%20Estudios/Highlights\%206\%BA\%20Observatorio\%20Final.p df (fecha de consulta: 19/07/10); ZENITH MEDIA (2011): «Panel Zenith Vigía sobre inversión publicitaria», disponible en www.zenithmedia.es (fecha de consulta: 19/08/11).

26 Grant, I. y O’DONOHOE, S. (2007): "Why young consumers are not open to mobile marketing communication», International Journal of Advertising, 26 (2), 223-246. 
anunciantes, tanto por su influencia en las compras familiares como por los patrones de fuerte consumismo que presentan.

Previo al desarrollo del estudio empírico, se llevaron a cabo tres dinámicas de grupo con jóvenes adolescentes, que ayudaron a (1) definir correctamente algunas de las preguntas formuladas en el cuestionario, así como las alternativas de respuesta y (2) plantear nuevas preguntas de interés. El cuestionario recogía tanto preguntas abiertas como cerradas, dicotómicas y de respuesta múltiple, así como preguntas de escalas, tipo likert de 5 puntos, todas ellas adaptadas de trabajos previos. El análisis de datos fue llevado a cabo utilizando el programa estadístico SPSS versión 17.0, permitiendo una descripción detallada del conocimiento, actitud, motivaciones de uso y valoración global de las aplicaciones publicitarias.

\section{Análisis de los resultados}

\section{Perfil de la muestra}

La muestra analizada se compone de un $58,5 \%$ de hombres y un $41,5 \%$ de mujeres con edades comprendidas entre los $14(30,9 \%), 15(34,6 \%)$ y $16(34,3 \%)$ años, siendo todos estudiantes de $2^{\circ}, 3^{\circ}$ y $4^{\circ}$ de E.S.O. (Tabla 1).

Tabla 1. Perfil de la muestra

\begin{tabular}{|c|l|c|}
\hline & \multicolumn{1}{|c|}{ Variables } & $\mathrm{N}=274$ \\
\hline \multirow{2}{*}{ Género } & Hombre & $58,5 \%$ \\
\cline { 2 - 3 } & Mujer & $41,5 \%$ \\
\hline \multirow{3}{*}{ Edad } & 14 años & $30,9 \%$ \\
\cline { 2 - 3 } & 15 años & $34,6 \%$ \\
\cline { 2 - 3 } & 16 años & $34,3 \%$ \\
\hline \multirow{3}{*}{ Curso } & $2^{\circ}$ ESO & $30,9 \%$ \\
\cline { 2 - 3 } & $3^{\circ} \mathrm{ESO}$ & $34,8 \%$ \\
\cline { 2 - 3 } & $4^{\circ} \mathrm{ESO}$ & $34,3 \%$ \\
\hline
\end{tabular}

Conocimiento de las aplicaciones publicitarias para móviles

Por lo que respecta al conocimiento de las aplicaciones publicitarias para móviles, un $34,3 \%$ de los encuestados afirmaron conocer dichas aplicaciones. No se detectaron diferencias significativas entre el porcentaje de hombres y el porcentaje de mujeres que manifestaron conocer las aplicaciones publicitarias para móviles. Tampoco se detectaron diferencias significativas entre los grupos segmentados por edades (14, 15 y 16 años). 


\section{Actitud hacia las aplicaciones publicitarias para móviles}

En general, la actitud de los jóvenes encuestados hacia las aplicaciones publicitarias para móviles es baja (Me gusta $=2,58$; Me parece interesante $=2,71$; Me parece una buena idea $=2,72$; Me parece algo positivo=2,67) en especial si se compara con la actitud que tienen hacia las aplicaciones para móviles que no son publicitarias (Me gusta=3,97; Me parece interesante $=4,03$; Me parece una buena idea $=4,25$; Me parece algo positivo $=$ $3,98)$. No obstante, resulta interesante destacar que esta actitud es mejor que la actitud hacia la publicidad móvil en general (Me gusta=1,50; Me parece interesante=1,53; Me parece una buena idea $=1,80$; Me parece algo positivo=1,70) (Tabla 2). No se detectaron diferencias significativas de la actitud hacia las aplicaciones publicitarias para móviles entre hombres y mujeres. Tampoco esta diferencia resulta significativa cuando es analizada por edades. Cuando se analizaron las actitudes (hacia la publicidad en general, hacia la publicidad móvil y hacia las aplicaciones para móvil) en función de si el usuario conocía o no las aplicaciones publicitarias para móvil, la prueba no paramétrica de MannWhitney (ver Tabla 3) tan sólo reveló diferencias significativas en relación a la actitud hacia la publicidad móvil. Una explicación a este hecho puede radicar en que quizás el usuario que conoce y utiliza las aplicaciones para móvil es un usuario más experto que realiza un uso más intensivo de su teléfono móvil y que, por tanto, es un usuario a quien le molesta más recibir comunicaciones publicitarias en ese medio (puesto que éstas quizás interrumpen sus actividades a través del teléfono móvil). El resto de variables presentaron valores muy similares entre ambos colectivos, siendo en general las actitudes bajas.

Tabla 2. Actitudes de los consumidores hacia la publicidad, hacia la publicidad móvil, hacia las aplicaciones para móvil y hacia las aplicaciones publicitarias para móvil.

\begin{tabular}{|c|c|c|c|}
\hline & & $\begin{array}{c}\text { SI } \\
\text { CONOCEN } \\
(\mathrm{N}=94)\end{array}$ & $\begin{array}{c}\text { NO } \\
\text { CONOCEN } \\
(\mathrm{N}=180)\end{array}$ \\
\hline \multirow{4}{*}{$\begin{array}{c}\text { ACTITUD } \\
\text { PUBLICIDAD }\end{array}$} & Me gusta & 2,78 & 2,70 \\
\hline & Me parece interesante & 2,89 & 2,66 \\
\hline & Me parece una buena idea & 3,31 & 2,94 \\
\hline & Me parece algo positivo & 2,84 & 2,71 \\
\hline \multirow{4}{*}{$\begin{array}{l}\text { ACTITUD } \\
\text { PUBLICIDAD } \\
\text { MOVIL }\end{array}$} & Me gusta & 1,50 & 1,68 \\
\hline & Me parece interesante & 1,53 & 1,74 \\
\hline & Me parece una buena idea & 1,80 & 2,00 \\
\hline & Me parece algo positivo & 1,70 & 1,91 \\
\hline \multirow{4}{*}{$\begin{array}{c}\text { ACTITUD } \\
\text { APLICACIONES }\end{array}$} & Me gusta & 3,97 & 2,30 \\
\hline & Me parece interesante & 4,03 & 2,00 \\
\hline & Me parece una buena idea & 4,25 & 2,50 \\
\hline & Me parece algo positivo & 3,98 & 2,45 \\
\hline \multirow{4}{*}{$\begin{array}{c}\text { ACTITUD } \\
\text { APLICACIONES } \\
\text { PUBLICITARIAS }\end{array}$} & Me gusta & 2,58 & No valorado \\
\hline & Me parece interesante & 2,71 & No valorado \\
\hline & Me parece una buena idea & 2,72 & No valorado \\
\hline & Me parece algo positivo & 2,67 & No valorado \\
\hline
\end{tabular}


Tabla 3. Diferencias significativas entre si conocen/no conocen las aplicaciones publicitarias

\begin{tabular}{|l|c|c|c|c|}
\hline & $\begin{array}{c}\text { TOTAL } \\
\text { N=274 }\end{array}$ & $\begin{array}{c}\text { SI } \\
\text { CONOCEN } \\
\text { N=94 }\end{array}$ & $\begin{array}{c}\text { NO } \\
\text { CONOCEN } \\
\text { N=180 }\end{array}$ & $\begin{array}{c}\text { Diferencias } \\
\text { Sig }\end{array}$ \\
\hline Actitud hacia la publicidad & 2,77 & 2,95 & 2,75 & $\begin{array}{c}6044,000 \\
(, 221)\end{array}$ \\
\hline Actitud hacia la publicidad móvil & 1,79 & 1,63 & 1,83 & $\begin{array}{c}5802,500 \\
(, 085)\end{array}$ \\
\hline Actitud hacia las aplicaciones & 3,93 & 4,05 & 3,90 & $\begin{array}{c}5989,500 \\
(, 183)\end{array}$ \\
\hline $\begin{array}{l}\text { Actitud hacia las aplicaciones } \\
\text { publicitarias }\end{array}$ & 2,67 & 2,67 & $\begin{array}{c}\text { No valora- } \\
\text { do }\end{array}$ & - \\
\hline
\end{tabular}

Motivos de uso de las aplicaciones publicitarias para móviles

El principal motivo de uso de las aplicaciones publicitarias para móviles es el entretenimiento. En especial en los hombres destaca el factor entretenimiento (Para entretenerme $=4,45$; Para divertirme $=4,43$; Para pasar el rato $=4,21$ ) muy por encima de los factores de autoimagen o incluso las recomendaciones de los amigos. En el caso de las mujeres es destacable, frente a los hombres, el factor curiosidad así como el factor prescriptivo de amistades y conocidos a la hora de descargarse este tipo de aplicaciones. Este hecho parece indicar que, por lo que respecta a las aplicaciones publicitarias para móviles, las mujeres se dejan influir. No obstante, las diferencias tan sólo son significativas en relación al factor curiosidad a un nivel de $\mathrm{p}<.01$ (Tabla 4).

Tabla 4. Motivos de uso de las aplicaciones publicitarias

\begin{tabular}{|l|c|c|c|c|}
\hline \multicolumn{1}{|c|}{ Motivos de uso } & $\begin{array}{c}\text { MEDIA } \\
(\mathrm{N}=94)\end{array}$ & $\begin{array}{c}\text { HOMBRES } \\
(\mathrm{N}=)\end{array}$ & $\begin{array}{c}\text { MUJERES } \\
(\mathrm{N}=)\end{array}$ & $\begin{array}{c}\text { Diferencias } \\
\text { Sig. }\end{array}$ \\
\hline 1. Por curiosidad & 2,92 & 2,65 & 3,50 & $\begin{array}{c}217,500 \\
(, 058)\end{array}$ \\
\hline 2. Para pasar el rato & 4,14 & 4,21 & 3,78 & $\begin{array}{c}240,500 \\
(, 138)\end{array}$ \\
\hline 3. Para divertirme & 4,39 & 4,43 & 3,78 & $\begin{array}{c}233,500 \\
(, 113)\end{array}$ \\
\hline 4. Para entretenerme & 4,43 & 4,45 & 3,92 & $\begin{array}{c}258,000 \\
(, 250)\end{array}$ \\
\hline 5. Para relajarme & 2,58 & 2,52 & 2,14 & $\begin{array}{c}276,000 \\
(, 399)\end{array}$ \\
\hline 6. Para estar a la moda & 2,21 & 2,13 & 2,35 & $\begin{array}{c}283,000 \\
(, 468)\end{array}$ \\
\hline 7. Para parecer una persona con estilo & 2,01 & 1,98 & 2,14 & $\begin{array}{c}289,000 \\
(, 529)\end{array}$ \\
\hline
\end{tabular}




\begin{tabular}{|l|c|c|c|c|}
\hline \multicolumn{1}{|c|}{ Motivos de uso } & $\begin{array}{c}\text { MEDIA } \\
(\mathrm{N}=94)\end{array}$ & $\begin{array}{c}\text { HOMBRES } \\
(\mathrm{N}=)\end{array}$ & $\begin{array}{c}\text { MUJERES } \\
(\mathrm{N}=)\end{array}$ & $\begin{array}{c}\text { Diferencias } \\
\text { Sig. }\end{array}$ \\
\hline 8. Para estar a la última & 2,23 & 2,00 & 2,64 & $\begin{array}{c}240,500 \\
(, 122)\end{array}$ \\
\hline 9. Porque me la recomendaron & 3,17 & 3,04 & 3,71 & $\begin{array}{c}231,500 \\
(, 103)\end{array}$ \\
\hline $\begin{array}{l}\text { 10. Porque mis amigos/conocidos la } \\
\text { tienen }\end{array}$ & 2,98 & 3,06 & 2,78 & $\begin{array}{c}283,500 \\
(, 490)\end{array}$ \\
\hline $\begin{array}{l}\text { 11. Porque recibí información a través } \\
\text { de publicidad }\end{array}$ & 1,84 & 1,84 & 2,00 & $\begin{array}{c}275,500 \\
(, 356)\end{array}$ \\
\hline
\end{tabular}

\section{Valoración de las aplicaciones publicitarias para móviles}

En general los encuestados realizan una valoración bastante neutra de las aplicaciones publicitarias para móviles (un 45,7\% de los hombres y un 64,3\% de las mujeres las valoran como "ni buenas ni malas"). Una posible explicación de esta valoración tan neutra de las aplicaciones publicitarias para móviles puede radicar en la novedad del formato para el consumidor. Dada su novedad, y la inexperiencia del consumidor con el formato, puede que el consumidor carezca todavía de un marco de referencia con el que comparar y emitir juicios de valor acerca del formato (que puede no ser percibido todavía como un formato publicitario pero que tampoco encaja del todo con otro tipo de aplicaciones para móviles más populares y conocidas por los consumidores). En todo caso, las mujeres parecen ser más críticas con el formato puesto que frente al $19,6 \%$ de los hombres que valoran las aplicaciones publicitarias para móviles como "muy malas", esta cifra se incrementa casi en 10 puntos en el caso de las mujeres $(28,6 \%)$ (Tabla 5). En todo caso, la prueba T para muestras independientes no muestra diferencias significativas en relación a la valoración de las aplicaciones publicitarias para móviles entre hombres y mujeres.

Tabla 5. Valoración de las aplicaciones publicitarias

\begin{tabular}{|l|c|c|c|c|}
\hline $\begin{array}{c}\text { En general, ¿cómo valoras las aplicacio- } \\
\text { nes publicitarias? }\end{array}$ & $\begin{array}{c}\text { MEDIA } \\
(\mathrm{N}=94)\end{array}$ & $\begin{array}{c}\text { HOMBRES } \\
(\mathrm{N}=)\end{array}$ & $\begin{array}{c}\text { MUJERES } \\
(\mathrm{N}=)\end{array}$ & $\begin{array}{c}\text { Diferencias } \\
\text { Sig. }\end{array}$ \\
\cline { 1 - 4 } Muy malas & $21,9 \%$ & $19,6 \%$ & $28,6 \%$ & 0,072 \\
\cline { 1 - 4 } Malas & $15,6 \%$ & $17,4 \%$ & $7,1 \%$ & \\
\cline { 1 - 4 } Ni buenas ni malas & $48,4 \%$ & $45,7 \%$ & $64,3 \%$ & \\
\cline { 1 - 4 } Buenas & $10,9 \%$ & $13,0 \%$ & $0 \%$ & \\
\hline Muy buenas & $3,1 \%$ & $4,3 \%$ & $0 \%$ & \\
\hline
\end{tabular}




\section{Conclusiones}

Las aplicaciones para móviles constituyen un mercado emergente en el sector de la telefonía móvil. Su capacidad para satisfacer necesidades de información, entretenimiento y socialización, las convierte además en un soporte prometedor para las comunicaciones publicitarias. No obstante los trabajos académicos que han analizado las actitudes de los consumidores hacia este tipo de formato publicitario (y asimismo cómo es percibido y valorado) son prácticamente inexistentes. En este sentido, el presente trabajo supone posiblemente uno de los primeros esfuerzos por conocer estos importantes factores utilizando una muestra de máximo interés para los anunciantes (adolescentes estudiantes de E.S.O.) debido al rol que desempeñan como usuarios y prescriptores de las aplicaciones. El análisis de los resultados revela que todavía existe un gran desconocimiento del formato por parte de los adolescentes españoles. Tan sólo un $34,3 \%$ de los encuestados afirmaron conocer dichas aplicaciones.

Con respecto a la actitud mostrada por los encuestados hacia las aplicaciones publicitarias para móviles hay que destacar que es baja. Este hecho es congruente con la valoración que realizan los encuestados de las aplicaciones publicitarias para móviles (en general, neutra, muy mala o mala). Parece evidente que los consumidores valoran de forma neutra o negativa las aplicaciones publicitarias para móviles con las que han interactuado y, como consecuencia, tienen una baja actitud no muy positiva hacia las mismas.

En cuanto a los motivos de descarga y uso de este tipo de aplicaciones por parte de los usuarios, destaca el factor entretenimiento. Este hecho es congruente con los motivos de uso de otros formatos publicitarios que también utilizan el entretenimiento como reclamo con el que atraer la atención del consumidor y primar su consumo (como los advergames o el branded content en general). En torno al factor de motivación existen algunas diferencias en función del género ya que en las mujeres es destacable el factor curiosidad así como el factor prescriptivo de amistades y conocidos a la hora de descargarse este tipo de aplicaciones.

\section{Implicaciones directivas, limitaciones y futuras líneas de investigación}

El auge de las aplicaciones para móviles supone un nuevo nicho comunicativo para las organizaciones dispuestas a innovar y a utilizar formatos publicitarios novedosos. Puesto que los consumidores que acceden a este tipo de aplicaciones publicitarias no representan el total del mercado, los anunciantes que incorporen este formato publicitario a sus herramientas de comunicación tienen la oportunidad de llegar a los nuevos consumidores que se incorporen al mercado a través de un formato novedoso en un contexto publicitario todavía no saturado.

Por otra parte, un hecho importante a destacar del estudio es que la actitud mostrada por los encuestados hacia las aplicaciones publicitarias para móviles es desfavorable. 
Puesto que la actitud hacia el anuncio (en este caso, la aplicación publicitaria para móvil) ejerce una importante influencia en la actitud hacia la marca emplazada en él, los resultados sugieren que los anunciantes deben mejorar las actitudes de los consumidores hacia las aplicaciones publicitarias para móvil que están desarrollando y lanzando al mercado. En este sentido, factores de diseño como el entretenimiento percibido por el usuario a través de la aplicación o las necesidades de información que satisfagan y los aspectos sociales que faciliten (comunicación e intercambio entre los usuarios) pueden ayudar a incrementar una actitud positiva de los consumidores.

Dada la naturaleza exploratoria de este estudio, una futura línea de investigación de interés estaría relacionada con la medición de la fuerza de la relación entre algunos factores que emergen de este estudio (por ejemplo, entre el factor entretenimiento y la actitud hacia las aplicaciones publicitarias para móviles). Asimismo, dado el relativo desconocimiento de los consumidores hacia este tipo de aplicaciones sería necesario indagar las causas de este desconocimiento al igual que la detección de aquellos motivos que impiden la descarga de estas aplicaciones: económicos (precio de las aplicaciones), técnicos (incompatibilidad de los terminales), personales (perfil más o menos innovador del consumidor), etc.

\section{Referencias bibliográficas}

AAKER, D. A., STAYMAn, D. M. Y VezINA, R. (1988): «Identifying Feelings Elicited by Advertising», Psychology and Marketing, 5 (primavera), 1-16.

ADELANTADO, E. Y MARTí, J. (2010): «Mobile advergames: tipología de la oferta en España y oportunidades de negocio». XXV Congreso Nacional de Comunicación: Modelo de negocio para una Economía digital: el valor de los contenidos. 25-26 noviembre, Pamplona, España. ASIMELEC. (2010): «Informe 2010 del macrosector TIC en España».

BATRA, R. Y RAY, M. L. (1986): «Affective Responses Mediating Acceptance of Advertising», Journal of Consumer Research, Vol. 13, (septiembre), 234-249.

CONECTA (2011): « $6^{\circ}$ observatorio de tendencias NOKIA: los jóvenes, los móviles y la tecnología», disponible en:

http://www.conectarc.com/Articulos\%20y\%20Estudios/Highlights\%206\%BA\%20Observat orio\%20Final.pdf (fecha de consulta: 19/07/10)

GARTNER (2009): «Dataquest Insight: Application Stores; The Revenue Opportunity Beyond the Hype», disponible en: http://www.gartner.com/resId=1257213 (fecha de consulta: 19/08/10)

Geuens, M. y De Pelsmacker, P. (1998): «Feelings Evoke by Warmth, Eroticism and Humour in Alcohol Advertissments», Journal of Consumer and Market Research, disponible en http://www.amsreview.org/articles/geuens01-1998.pdf (fecha de consulta: 29/07/07)

GrANT, I. Y O'DONOHOE, S. (2007): «Why young consumers are not open to mobile marketing communication», International Journal of Advertising, 26 (2), 223-246.

HAGGIRIAN, P., MADLBERGER, M. Y TANUSKOVA, A. (2005): «Increasing Advertising Value of Mobile Marketing- An Empirical Study of Antecedents», Proceedings of the 38th Hawaii International Conference on System Sciences. 3-6 enero, Hawaii. 
INTERACTIVE ADVERTISING BUREAU (IAB) (2009): «Estudio de Internet e interactividad en móviles y otros dispositivos portátiles», disponible en: http://www.iabspain.net/ver.php?mod=noticias\&identificador $=40$ (fecha de consulta: $09 / 05 / 10)$

INTERNATIONAL TELECOMMUNICATION UNION (2011): «The World in 2011: ICT Facts and Figures», disponible en: http://www.itu.int/ITU-D/ict/material/FactsFigures2011.pdf (fecha de consulta: 30/07/11).

KARGIN, B., NURI BASOGLU, N. Y TUGRUL DAIM, T. (2009): «Factors affecting the adoption of mobile services», International Journal of Services Sciences, Vol. 2, №1, pp. 29-52.

KATZ, E., HAAS, H. y GUREVITCH, M. (1973): «On the Use of the Mass Media for Important Things», American Sociological Review, 38 (2), 164-181.

Kim, J. AllEN, C. T Y KARDES, F. R. (1996): «An Investigation of the Mediational Mechanisms Underlying Attitudinal Conditioning», Journal of Marketing Research, 33 (agosto), 318328.

LAVIDGE, R. J. y SteINER, G. A. (1961): «A Model for Predictive Measurements of Advertising Effectiveness», Journal of Marketing, 24, 59-62.

LEPPÄNIEMI, M., SINISALO, J. Y KARJALUOTO, H. (2006): «A review of mobile marketing research, International Journal of Mobile Marketing», 1 (1), 30-40.

LuTZ, R.J. (1985): «Affective and Cognitive Antecedents of Attitude Toward the Ad: A Conceptual Framework», Alwitt, L. y Mitchell, A. (Eds.), Psychological Processes and Advertising Effects, Hillsdale (NJ), Lawrence J. Eribaum, 45-65.

MACKENZIE, S.C., LutZ, R. J. Y BELCH, G. E. (1986): «The Role of Attitude toward the Ad as a mediator of Advertising Effectiveness: A Test of Competing Explanations», Journal of Marketing Research, 23, (mayo), 130-143.

MCGuIRE, W. J. (1978): «An Information Processing Model of Advertising Effectiveness», en Davis, H.L y Silk, A.J. (eds.) Behavioral and Management Science in Marketing, Nueva York, Ronald/Wiley.

Mitchell, A. A. Y OlSON, J. C. (1981): «Are Product Attribute Beliefs the Only Mediator of Advertising Effects on Brand Attribute?», Journal of Marketing Research, 18, (agosto), 318332.

MMA (Mobile MARKeting Association) (2008): «Mobile Applications», disponible en: http://mmaglobal.com/mobileapplications.pdf (fecha de consulta: 14/07/09)

MMA (MOBILE MARKeting Association) (2010): «3er Estudio de Inversión en Marketing y Publicidad Móvil en España 2010», disponible en:

http://recursos.anuncios.com/files/387/64.pdf (fecha de consulta: 19/04/11)

MoORE, D. L. Y Hutchinson, J. W. (1983): «The Effects of Ad Affect on Advertising Effectiveness», en Bagozzi, R. y Tybout, A. (eds.), Advances in Consumer Research, vol. 10, Ann Arbor (Michigan), Association for Consumer Research, 526-531.

OKAZAKI, S., LI, H. Y HIROSE, M. (2009): «Consumer privacy concerns and preference for degree of regulatory control: A study of mobile advertising in Japan», Journal of Advertising, 38 (4), 63-77.

PARK, C. W. Y Young, S. M. (1986): «Consumer Response to Television Commercials: The Impact of Involvement and Background Music on Brand Attitude Formation», Journal of Marketing Research, 23, (febrero), 11-24. 
PQ MEDIA (2008): «Alternative media forecast: 2008-2012», disponible en: http://www.pqmedia.com/alternative-media-forecast-2008.html (fecha de consulta: $12 / 05 / 09)$

SANZ, S., RUIZ, C. Y MARTí, J. (2011): «Situación actual de las comunicaciones de marketing mediante telefonía móvil», ponencia presentada en el XXV Congreso Anual de la Academia Europea de Economía y Dirección de Empresa, Actas del Congreso, Valencia 8, 9 y 10 de junio.

SCHARL, A., Dickinger, A. Y MURPHY, J. (2005): «Diffusion and success factors of mobile marketing», Electronic Commerce Research and Applications, 4 (2), 159-173.

Shankar, V. y Balasubramanian, S. (2009): «Mobile Marketing: A Synthesis and Prognosis», Journal of Interactive Marketing, 23, 118-129.

SHIMP, T. A. (1981): «Attitude toward the Ad as Mediator of Consumer Brand Choice», Journal of Advertising, 10 (2), 9-15.

STRONG, E. K. (1925): The Psychology of Selling, Nueva York, MacGraw-Hill.

TSANG, M.M., HO, S-C. Y LIANG, T-P. (2004): «Consumer attitudes toward mobile advertising: An empirical study», International Journal of Electronic Commerce, 8 (3), 65-78.

WALDT, REBBeLlo, T.M. Y BROWN W.J. (2009): «Attitude of Young Consumers toward SMS", African Journal of Business Management», 3 (9), 444-452.

XU, D.J.J. (2006): «The influence of personalization in affecting consumer attitudes toward mobile advertising in China», Journal of Computer Information Systems, 47 (2), 9-19.

XU, D.J., LIAO, S.S. Y LI, Q. (2008): «Combining empirical experimentation and modelling techniques: A design research approach for personalized mobile advertising applications», Decision Support Systems, 44 (3), 710-724.

ZENITH Media (2011): «Panel Zenith Vigía sobre inversión publicitaria», disponible en www.zenithmedia.es (fecha de consulta: 19/08/11)

ZHANG, J. Y MAO, E. (2008): «Understanding the Acceptance of Mobile SMS Advertising among Young Chinese Consumers», Psychology \& Marketing, 25 (8), 787-805.

Recibido: 2 de diciembre de 2011

Aceptado: 18 de enero 2012 